\title{
Patterns of recurrence and survival in sporadic, neurofibromatosis Type 1-associated, and radiation-associated malignant peripheral nerve sheath tumors
}

\author{
Kelsey L. Watson, BS, ${ }^{1}$ Ghadah A. AI Sannaa, MD, ${ }^{2}$ Christine M. Kivlin, BS, ${ }^{1,3}$ \\ Davis R. Ingram, BS, ${ }^{2}$ Sharon M. Landers, PhD, ${ }^{1}$ Christina L. Roland, MD, ${ }^{1}$ \\ Janice N. Cormier, MD, MPH, ${ }^{1}$ Kelly K. Hunt, MD, ${ }^{1}$ Barry W. Feig, MD, ${ }^{1}$ B. \\ Ashleigh Guadagnolo, MD, MPH, ${ }^{4}$ Andrew J. Bishop, MD, ${ }^{4}$ Wei-Lien Wang, MD, ${ }^{2}$ \\ John M. Slopis, MD, ${ }^{5}$ lan E. McCutcheon, MD, ${ }^{6}$ Alexandar J. Lazar, MD, PhD, ${ }^{3}$ and \\ Keila E. Torres, MD, PhD'
}

Departments of ${ }^{1}$ Surgical Oncology, ${ }^{2}$ Pathology, ${ }^{4}$ Radiation Oncology, ${ }^{5}$ Neuro-Oncology, and ${ }^{6}$ Neurosurgery, The University of Texas MD Anderson Cancer Center; and ${ }^{3}$ The University of Texas Health Science Center at Houston Graduate School of Biomedical Sciences, Houston, Texas

\begin{abstract}
OBJECTIVE Malignant peripheral nerve sheath tumors (MPNSTs) are an aggressive group of soft tissue sarcomas that can arise sporadically, in the context of neurofibromatosis Type 1 (NF1) or at a site of prior irradiation. Large series profiling the features and outcomes of sporadic, NF1-associated, and radiation-associated MPNSTs are limited. The goal of this study was to elucidate differences between MPNST etiologies in a large single-institution retrospective study.

METHODS Patients $(n=317)$ were identified through the tumor registry of The University of Texas MD Anderson Cancer Center. Clinicopathological features were retrospectively collected. Features were compared among MPNST subtypes for patients who had sufficient clinical history $(n=289)$, and clinicopathological features were used to identify adverse predictors of recurrence and survival outcomes.

RESULTS Five-year local recurrence-free survival (LRFS), distant recurrence-free survival (DRFS), and diseasespecific survival (DSS) estimates were $56.6 \%, 49.6 \%$, and $53.6 \%$, respectively, for the high-grade MPNST cohort. Fiveyear DSS was lower in NF1-associated and radiation-associated MPNST than in sporadic MPNST $(52 \%, 47 \%$, and $67 \%$, respectively, $p=0.140$ ). Patients with radiation-associated MPNST had worse 5-year LRFS than those with the sporadic and NF1-associated subtypes (RT-associated vs sporadic, $p=0.010$; RT-associated vs NF1-associated, $p=0.232$ ). Truncally located tumors, positive surgical margins, local recurrence, and metastasis were predictors of adverse DSS in multivariate analysis.
\end{abstract}

CONCLUSIONS Radiation-associated MPNSTs are associated with poorer local recurrence-free and disease-specific survival than sporadic and NF1-associated tumors. NF1-associated MPNSTs may have worse survival outcomes owing to large tumor size, compromising truncal location, and lower rate of negative resection margins compared with sporadic tumors.

http://thejns.org/doi/abs/10.3171/2015.12.JNS152443

KEY WORDS malignant peripheral nerve sheath tumor; MPNST; neurofibromatosis Type 1; radiation-induced sarcoma

$\mathrm{A}$ CCOUNTING for $2 \%-5 \%$ of soft tissue sarcomas, malignant peripheral nerve sheath tumors (MPNSTs) are a complex group of tumors that arise from the peripheral nerve sheath. ${ }^{4,9}$ Approximately 40\%-50\% of MPNSTs arise within the setting of neurofibromatosis Type 1 (NF1), a highly penetrant autosomal dominant ge- netic disorder caused by a loss-of-function mutation in the $N F 1$ gene. The lifetime incidence of malignant transformation of existing neurofibromas in NF1 patients is approximately $10 \% .8,24$ An additional $40 \%-47 \%$ of MPNSTs develop sporadically, and $10 \%-13 \%$ arise in a prior field of therapeutic radiation. ${ }^{8,15}$ Management of these tumors is

ABBREVIATIONS DRFS = distant recurrence-free survival; DSS = disease-specific survival; HR = hazard ratio; LRFS = local recurrence-free survival; MPNST = malignant peripheral nerve sheath tumor; NF1 = neurofibromatosis Type 1.

SUBMITTED October 21, 2015. ACCEPTED December 11, 2015.

INCLUDE WHEN CITING Published online April 1, 2016; DOI: 10.3171/2015.12.JNS152443. 
challenging, as the benefit of chemotherapy has not been widely demonstrated, and the success of radiotherapy for local control has not consistently been reported. , $^{1,7,19,23,25}$ Negative margin resection-often impeded by large tumor size and extensive nerve involvement-remains the mainstay of curative treatment. ${ }^{3,11}$ MPNSTs have a high propensity for local relapse without complete resection and a high risk for metastatic spread. Prognosis in patients with MPNST remains poor, with reported 5-year disease-specific survival (DSS) rates ranging from 39\% to $60 \%$ in multiple single-institution series over the last 15 years. ${ }^{1,7,19,23,25}$ However, some small studies have demonstrated superior survival in patients with low-grade variants of MPNST, suggesting a benign natural history in these rare lesions compared with their high-grade counterparts. ${ }^{2,22}$ Whether NF1 is adversely associated with survival remains controversial..$^{14,19,23}$ Additionally, studies comparing outcomes of radiation-associated MPNST to other subtypes are sparse as a consequence of their rarity. ${ }^{15}$ The objective of this study was to identify adverse predictors of recurrence and survival in patients with sporadic, NF1-associated, and radiation-associated MPNSTs. As a secondary goal, low-grade MPNSTs were interrogated separately from high-grade MPNSTs to determine their natural history and optimal clinical management.

\section{Methods}

With approval of The University of Texas MD Anderson Cancer Center Institutional Review Board, a retrospective database containing 317 patients with a pathologically confirmed MPNST diagnosis between 1990 and 2014 was constructed. Data were collected under a Waiver of Consent/Authorization approved by the institutional review board. Patients who had adequate clinical history (n = 289) were included for statistical analysis (Fig. 1). Clinicopathological characteristics were collected in a comprehensive medical record review. A diagnosis of sporadic MPNST was made as previously described and in the absence of NF1 or prior local radiotherapy for a different malignancy. ${ }^{25}$ A tumor was classified as NF1-associated if documented genetic testing confirmed a germline NF1 mutation or by clinical evaluation based on the $1987 \mathrm{Na}-$ tional Institutes of Health consensus criteria for diagnosis of NF1. ${ }^{18}$ Patients with a history of therapeutic radiotherapy at least 6 months prior for an unrelated malignancy within the local field containing the newly arising MPNST were considered to have a radiation-associated MPNST. ${ }^{10}$

Resection margins were obtained from pathology notes. A resection margin was considered microscopically negative (R0) if the closest margin was $>1 \mathrm{~mm}$ from the inked surface, microscopically positive (R1) if the closest margin was $\leq 1 \mathrm{~mm}$ from the inked surface, or macroscopically positive (R2) for any subtotal resection where gross disease was present at inked margins. Tumors were graded as high or low on the basis of nuclear atypia, presence of hyperchromasia, mitotic activity, cellularity, and growth pattern. ${ }^{6,21}$ Select studies have demonstrated more favorable outcomes in low-grade MPNSTs compared with high-grade MPNSTs; therefore, we reviewed patients with low-grade MPNSTs independently $(\mathrm{n}=12)^{2,22}$ Patients in the high-grade cohort who did not receive surgical intervention as part of their primary treatment $(n=22)$ or who underwent subtotal $\mathrm{R} 2$ resection $(\mathrm{n}=23)$ were evaluated independently, owing to their anticipated poor prognosis. Only patients who had primary tumors in the absence of synchronous metastasis at diagnosis were included in the outcomes analysis (Fig. 1).

Characteristics between MPNST subtypes were compared using chi-square or Fisher's exact test for categorical variables and the nonparametric Mann-Whitney U-test or Kruskal-Wallis test for continuous variables, as appropriate. Log-rank methods were employed to estimate local recurrence-free survival (LRFS), distant recurrence-free survival (DRFS), and DSS outcomes. A disease-specific event was recorded if the patient experienced MPNSTspecific death. Multivariate Cox regression models were constructed by including any variables with statistical significance below a $\mathrm{p}$ value cutoff of 0.05 at the univariate level. All 2 -sided $p$ values $<0.05$ were considered statistically significant. All computations were performed using SPSS version 22.0 (IBM Corp.).

\section{Results}

\section{Patient Characteristics and Presentation}

A total of 289 patients with MPNST treated at our institution between 1990 and 2014 were included. Tumor and demographic characteristics of these patients are summarized in Table 1 . The median duration of follow-up was 2.13 years (range $0.05-36.0$ years), 2.18 years (range $0.03-$ 29.8 years), and 1.74 years (range $0.25-13.7$ years) for sporadic, NF1-associated, and radiation-associated MPNSTs, respectively. One hundred fifteen patients (40\%) presented with sporadic disease, 148 (51\%) with MPNST in association with NF1, and 26 (9\%) with MPNST subsequent to previous local radiation therapy for a different malignancy. The median latency period for the development of radiation-associated MPNST from prior irradiation was 16 years (range $0.86-37$ years); the most common indications for prior radiotherapy were breast carcinoma (33\%) and Hodgkin's lymphoma (22\%).

The diagnosis of MPNST was established histologically in all cases (MPNST 89\%, epithelioid MPNST 7\%, triton tumor 4\%). Primary disease was most commonly located in the trunk (54\%), followed by the extremities (31\%), but location varied between MPNST subtypes ( $\mathrm{p}$ $=0.008$ ). The most commonly affected nerve in the 157 cases of truncally located disease was within the lumbosacral nerve roots $(n=37$ [24\%]), followed by the brachial plexus $(n=30[19 \%])$ and the thoracic nerve roots $(n=17$ [11\%]). The sciatic nerve was affected in $12(8 \%)$ of 157 truncally located cases and $25(40 \%)$ of 63 lower extremity cases; 19 (50\%) of 38 cases of NF1-associated MPNSTs located within the lower extremity arose from the sciatic nerve. Symptoms at primary diagnosis were available for 245 patients (85\%); 38 patients (16\%) were asymptomatic but with a palpable mass effect, and 4 patients $(2 \%)$ were asymptomatic with incidental discovery of their disease. Two hundred and three (83\%) of 245 patients were symptomatic, and 120 (49\%) of these 245 patients reported more than 1 symptom. The most common symptom was 


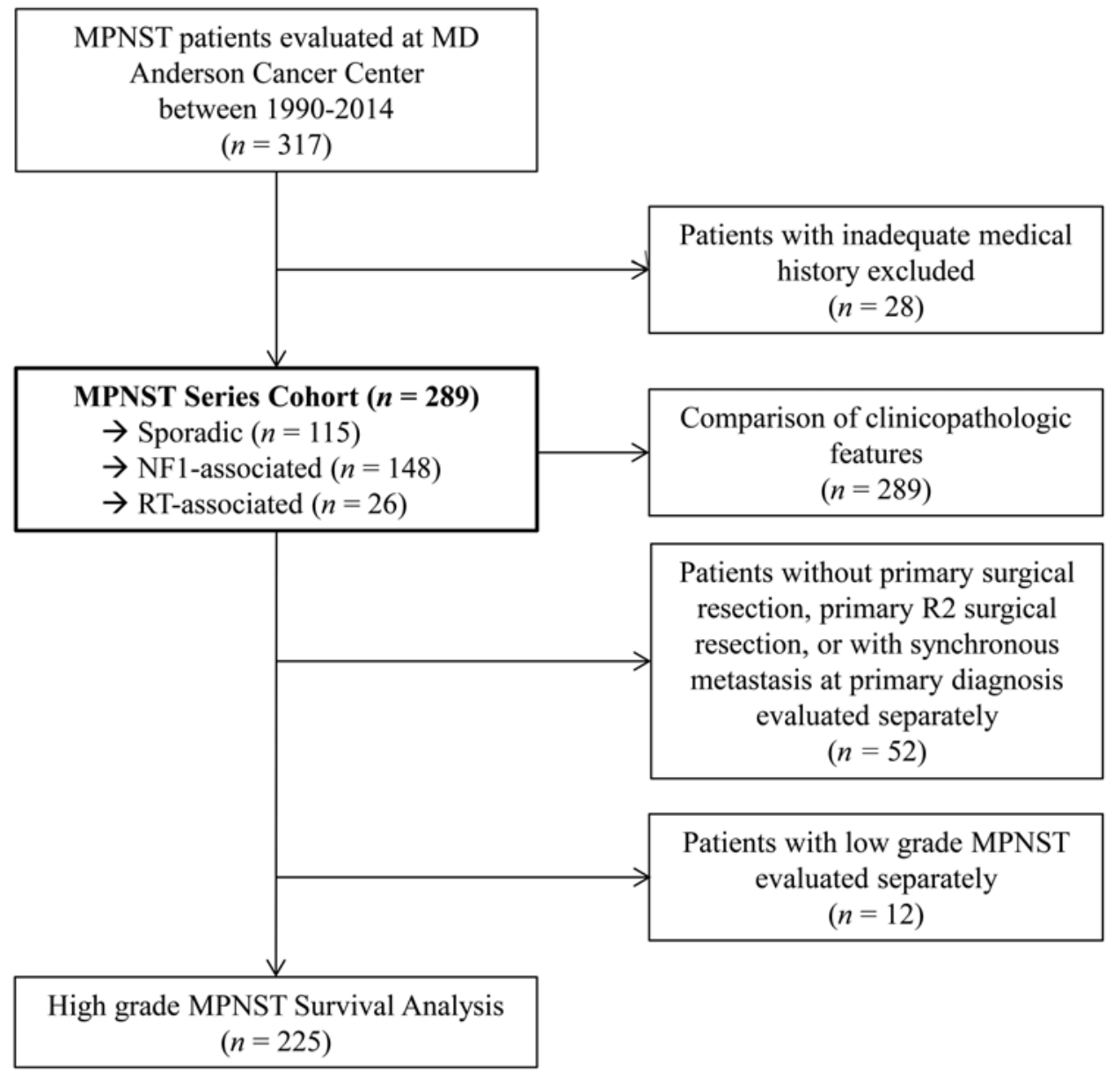

FIG. 1. Flowchart showing patient selection and study analysis.

pain in $173(71 \%)$ patients, followed by extremity weakness in $32(13 \%)$, extremity numbness in $22(9 \%)$, paresthesia in $14(6 \%)$, and gait instability or foot drop in 13 $(5 \%)$. There were no statistically significant differences between symptoms at presentation and MPNST subtype; however, patients with NF1-associated tumors were more likely to be symptomatic at presentation than patients with sporadic or radiation-associated tumors $(90 \%$ vs $75 \%$ and $73 \%$, respectively, $\mathrm{p}=0.006$ ).

Twelve tumors (4\%) were determined to be low grade. Low-grade MPNSTs were more likely to be superficially located compared with high-grade MPNSTs (31\% vs $5 \%$ for low-grade and high-grade MPNSTs, respectively, $p$ $=0.004)$. All NF1-associated low-grade MPNSTs arose within a neurofibroma.

\section{Treatment Characteristics}

Two hundred sixty-seven patients (92\%) underwent resection of the primary disease. One hundred fifty-nine of these patients (59\%) underwent definitive resection of their primary tumor at outside institutions, and 108 (41\%) had their tumor excised at our institution. The margin status was reviewed by a sarcoma pathologist at our institution. Resection was not performed in the remaining cases due to advanced disease $(n=17)$ or medical comorbidities $(n=5)$.
Nine patients (3\%) with localized primary tumors required amputation, and an additional 6 patients $(2 \%)$ required amputation after local recurrence. Twenty-three patients (8\%) underwent R2 resections (Table 2). Of these procedures, 17 were carried out with curative intent; however, additional resection to obtain negative margins was not pursued owing to rapidly progressive disease $(n=9)$ or prohibitive location $(\mathrm{n}=8)$. Median time to disease-specific death following incomplete (R2) resection was 0.8 years. Six patients underwent palliative R2 resection. Indications for palliative resection were pain ( $\mathrm{n}=3$ ), spinal cord compression $(n=2)$, and bowel obstruction $(n=1)$. Five of 6 patients reported temporary alleviation of symptoms; however, all patients had progressive disease, resulting in death following palliative procedures. Median time to disease-specific death following palliative $(\mathrm{R} 2)$ resection was 1.19 years.

Two hundred seventy-four patients $(95 \%)$ presented with localized disease; $75(27 \%)$ of these 274 patients underwent resection alone (Table 2). In addition to resection, 45 patients (16\%) received chemotherapy, 75 (27\%) received radiation therapy, $64(24 \%)$ received combination chemotherapy and radiation therapy, and $15(5 \%)$ underwent palliative chemotherapy or radiotherapy without resection. Chemotherapy regimens varied but most often included doxorubicin \pm ifosfamide as a first-line therapy; 
TABLE 1. Demographic and tumor characteristics of 289 patients with MPNSTs*

\begin{tabular}{|c|c|c|c|c|c|}
\hline \multirow[b]{2}{*}{ Characteristic } & \multicolumn{4}{|c|}{ MPNST } & \multirow[b]{2}{*}{ p Valuest } \\
\hline & $\begin{array}{l}\text { Overall, } n=289 \\
(100)\end{array}$ & $\begin{array}{c}\text { Sporadic, } n=115 \\
(39.8)\end{array}$ & $\begin{array}{c}\text { NF1-Assoc, } \\
\mathrm{n}=148(51.2)\end{array}$ & $\begin{array}{l}\text { Radiation-Assoc, } \\
n=26(8.9)\end{array}$ & \\
\hline Age in years & & & & & $<0.001(<0.001)$ \\
\hline Median & 37 & 43 & 33 & 52 & \\
\hline Range & $1-81$ & $5-81$ & $1-75$ & $20-77$ & \\
\hline Tumor size in $\mathrm{cm}$ & & & & & $<0.001(<0.001)$ \\
\hline Median & 8.0 & 6.0 & 10 & 6.0 & \\
\hline Range & $1.0-35.0$ & $1.0-32.0$ & $2.5-35.0$ & $1.5-15.0$ & \\
\hline Sex & & & & & $0.720(0.906)$ \\
\hline $\mathrm{F}$ & $134(46.4)$ & $52(45.2)$ & $68(45.9)$ & $14(53.8)$ & \\
\hline M & $155(53.6)$ & $63(54.8)$ & $80(54.1)$ & $12(46.2)$ & \\
\hline \multicolumn{6}{|l|}{ Disease at primary Dx } \\
\hline Localized & $274(94.8)$ & $105(91.3)$ & $143(96.6)$ & $26(100.0)$ & $0.071(0.065)$ \\
\hline Metastatic & $15(5.2)$ & $10(8.7)$ & $5(3.4)$ & $0(0.0)$ & \\
\hline \multicolumn{6}{|l|}{ Location } \\
\hline Head/neck & $43(14.9)$ & $23(20.0)$ & $13(8.8)$ & $7(26.9)$ & $0.008(0.211) \ddagger$ \\
\hline Cervical nerve root & $15(5.2)$ & $4(3.5)$ & $5(3.4)$ & $6(23.0)$ & \\
\hline Cranial nerve & $13(4.5)$ & $8(6.9)$ & $4(2.7)$ & $1(3.8)$ & \\
\hline Other nerve & $9(3.1)$ & $8(6.9)$ & $1(0.7)$ & $0(0.0)$ & \\
\hline Nerve unknown & $6(2.1)$ & $3(2.6)$ & $3(2.0)$ & $0(0.0)$ & \\
\hline Trunk & $157(54.3)$ & $54(47.0)$ & $85(57.4)$ & $18(69.2)$ & \\
\hline Brachial plexus trunks/divisions & $21(7.3)$ & $5(4.3)$ & $9(6.1)$ & $9(34.6)$ & \\
\hline Brachial plexus cords & $9(3.1)$ & $4(3.5)$ & $2(1.3)$ & $3(11.5)$ & \\
\hline Lumbosacral nerve root & $37(12.8)$ & $19(16.5)$ & $18(12.2)$ & $0(0.0)$ & \\
\hline Thoracic nerve root & $17(5.9)$ & $3(2.6)$ & $12(8.1)$ & $2(7.6)$ & \\
\hline Sciatic nerve & $12(4.2)$ & $1(0.8)$ & $10(6.8)$ & $1(3.8)$ & \\
\hline Phrenic nerve & $4(1.4)$ & $1(0.8)$ & $3(2.0)$ & $0(0.0)$ & \\
\hline Obturator nerve & $4(1.4)$ & $0(0.0)$ & $4(2.7)$ & $0(0.0)$ & \\
\hline Femoral nerve & $6(2.7)$ & $2(1.7)$ & $3(2.0)$ & $1(5.9)$ & \\
\hline Other nerve & $25(8.7)$ & $7(6.1)$ & $16(10.8)$ & $2(11.8)$ & \\
\hline Nerve unknown & $20(6.9)$ & $12(10.4)$ & $8(5.4)$ & $0(0.0)$ & \\
\hline Upper-extremity nerve & $26(8.9)$ & $13(11.3)$ & $12(8.1)$ & $1(3.8)$ & \\
\hline Radial & $4(1.4)$ & $2(1.7)$ & $2(1.3)$ & $0(0.0)$ & \\
\hline Median & $7(2.4)$ & $3(2.6)$ & $3(2.0)$ & $1(3.8)$ & \\
\hline Ulnar & $7(2.4)$ & $3(2.6)$ & $4(2.7)$ & $0(0.0)$ & \\
\hline Other & $4(1.4)$ & $3(2.6)$ & $1(0.6)$ & $0(0.0)$ & \\
\hline Nerve unknown & $4(1.4)$ & $2(1.7)$ & $2(1.3)$ & $0(0.0)$ & \\
\hline Lower-extremity nerve & $63(21.8)$ & $25(21.7)$ & $38(25.7)$ & $0(0.0)$ & \\
\hline Sciatic & $25(8.7)$ & $6(5.2)$ & $19(12.8)$ & $0(0.0)$ & \\
\hline Femoral & $7(2.4)$ & $3(2.6)$ & $4(1.4)$ & $0(0.0)$ & \\
\hline Obturator & $2(0.7)$ & $0(0.0)$ & $2(1.3)$ & $0(0.0)$ & \\
\hline Tibial & $9(3.1)$ & $5(4.3)$ & $4(2.7)$ & $0(0.0)$ & \\
\hline Other & $10(3.5)$ & $3(2.6)$ & $6(4.1)$ & $0(0.0)$ & \\
\hline Nerve unknown & $10(3.5)$ & $8(6.9)$ & $2(1.3)$ & $0(0.0)$ & \\
\hline \multicolumn{6}{|l|}{ Depth§ } \\
\hline D & $272(94.1)$ & $104(90.4)$ & $143(96.6)$ & $25(96.2)$ & $0.096(0.037)$ \\
\hline$S$ & $17(5.9)$ & $11(9.6)$ & $5(3.4)$ & $1(3.8)$ & \\
\hline
\end{tabular}


TABLE 1. Demographic and tumor characteristics of 289 patients with MPNSTs*

\begin{tabular}{|c|c|c|c|c|c|}
\hline \multirow[b]{2}{*}{ Characteristic } & \multicolumn{4}{|c|}{ MPNST } & \multirow[b]{2}{*}{ p Valuest } \\
\hline & $\begin{array}{c}\text { Overall, } n=289 \\
(100)\end{array}$ & $\begin{array}{c}\text { Sporadic, } n=115 \\
(39.8)\end{array}$ & $\begin{array}{c}\text { NF1-Assoc, } \\
n=148(51.2)\end{array}$ & $\begin{array}{l}\text { Radiation-Assoc, } \\
\quad n=26(8.9)\end{array}$ & \\
\hline MPNST, not further classified & $258(89.3)$ & $94(81.7)$ & $140(94.6)$ & $24(92.3)$ & $0.001(<0.001)$ \\
\hline Epithelioid & $20(6.9)$ & $17(14.8)$ & $2(1.4)$ & $1(3.8)$ & \\
\hline Triton tumor & $11(3.8)$ & $4(3.5)$ & $6(4.1)$ & $1(3.8)$ & \\
\hline Low-intermediate & $12(4.2)$ & $6(5.2)$ & $5(3.4)$ & $1(3.8)$ & $0.791(0.480)$ \\
\hline High (or not evaluated) & $277(95.8)$ & $109(94.8)$ & $143(96.6)$ & $25(96.2)$ & \\
\hline
\end{tabular}

Assoc $=$ associated $;$ chemo $=$ chemotherapy $; D=$ deep; $D x=$ diagnosis $; S=$ superficial.

* Values are number (\%) unless otherwise indicated. The sum of percentages may not equal 100 due to rounding.

$\dagger$ In each cell, the first value is for comparison of sporadic, NF1-associated, and radiation-associated characteristics; the second value is for comparison of sporadic and NF1-associated characteristics. Boldface type indicates statistical significance of $p<0.05$.

$\ddagger$ Additionally, comparisons were made between 4 major anatomical locations: head/neck, trunk, upper extremity, lower extremity.

$\S$ Tumors with extension through or seated below the superficial fascia were considered deep.

others included gemcitabine \pm docetaxel, cisplatin, dacarbazine, cyclophosphamide, epirubicin, vincristine, etoposide, and pazopanib.

Of 15 patients $(5 \%)$ with metastasis at initial presentation, 7 (2\%) received systemic therapy, 4 (1\%) underwent chemotherapy in addition to resection, and 4 (1\%) underwent combination chemoradiotherapy in addition to resection. Review of the patients with metastatic disease who underwent surgery revealed that 2 patients required surgical intervention to provide palliation. One patient presented with intense neck pain and the second patient had urinary and bowel obstruction. Six patients underwent resection at outside hospitals; 5 of whom had staging imaging at the time of surgery and were known to have metastatic disease. The median survival of patients with metastatic disease who underwent surgery was 0.78 years versus 1.73 years for those who were treated with systemic therapy.

\section{Low-Grade MPNST Outcomes}

Only $3(25 \%)$ of 12 patients with low-grade MPNST developed local recurrence after resection (Table 3). R0 resection was achieved in 1 of 3 recurring cases and 7 of 9 nonrecurring cases. No patients with low-grade lesions developed metastasis, and 5-year DSS outcomes were superior to all high-grade cohort outcomes, with $100 \%$ of patients surviving at the end of the study period (Fig. 2).

\section{High-Grade MPNST Local and Distant Recurrence-Free Survival}

Local and distant recurrence outcomes were assessed for 225 patients (sporadic, $\mathrm{n}=89$; NF1-associated, $\mathrm{n}=119$; radiation-associated, $\mathrm{n}=17$ ) who presented with highgrade localized disease and had undergone R0 or R1 resection. After a median follow-up of 2.7 years (range $0.03-36.0$ years), 84 patients (37\%) developed local recurrence (34\%, $38 \%$, and $53 \%$ for sporadic, NF1-associated, and radiationassociated subtypes, respectively) after resection. Median time to local recurrence was 0.95 years. Nine patients un- derwent amputation; 3 patients experienced recurrence at the stump. Of the 84 patients $(37 \%)$ who experienced local recurrence, 18 (21\%) underwent resection alone for recurrent disease, $45(42 \%)$ received chemotherapy or radiotherapy in addition to resection, and 21 (9.3\%) did not receive salvage intervention. A comparison of patients who underwent resection at our institution versus outside institutions did not reveal any statistically significant difference in LRFS (3-year LRFS following R0 resection 84\% vs $77 \%$, respectively, $\mathrm{p}=0.286$; 3 -year LRFS following R1 resection $48 \%$ vs $38 \%$, respectively, $\mathrm{p}=0.425$ ).

One hundred and five patients (47\%) developed distant recurrence $(47 \%, 47 \%$, and $41 \%$ for sporadic, NF1-associated, and radiation-associated subtypes, respectively). Median time to distant recurrence was 1.4 years. Thirty-three (31\%) of 105 patients had metastases to multiple organs; the most common locations included the lungs $(n=85)$, paraspinal region $(n=12)$, bone $(n=10)$, lymph nodes $(n=$ $8)$, brain $(n=7)$, liver $(n=5)$, pelvis $(n=5)$, and leptomeningeal $(n=4)$. Less common sites of metastases involved the mediastinum, retroperitoneum, musculature of the extremities, bladder, vagina, and spleen.

Univariate analyses for LRFS and DRFS revealed no significant differences in outcomes between neoadjuvant and adjuvant radiation therapy or chemotherapy. Therefore, neoadjuvant and adjuvant therapy variables were combined to strengthen statistical power (Table 4). The 5 -year LRFS was 59\%. Univariate analysis revealed that patients with sporadic tumors had better LRFS compared with patients with radiation-associated tumors $(\mathrm{p}=0.010$, hazard ratio [HR] 0.42; Table 4, Fig. 3). Patients who received radiation therapy in addition to resection had a better LRFS compared with patients who did not receive radiation therapy $(p=0.040$, HR 0.64 ; Table 4$)$. In multivariate analysis, tumors $\geq 10 \mathrm{~cm}$ and with positive margins remained significant prognosticators of adverse LRFS ( $p=$ 0.056, HR 2.09; $<$ < 0.001 HR 3.13, respectively; Table 4).

At our institution, radiotherapy is typically not offered to patients who present with radiation-associated MPNST, 
TABLE 2. Treatment characteristics of 274 patients with localized disease at presentation*

\begin{tabular}{|c|c|c|c|c|c|}
\hline \multirow[b]{2}{*}{ Characteristic } & \multicolumn{4}{|c|}{ MPNST } & \multirow[b]{2}{*}{$\begin{array}{l}p \text { Value } \\
\text { (p Value) } \dagger\end{array}$} \\
\hline & $\begin{array}{c}\text { Overall, } n=274 \\
(100)\end{array}$ & $\begin{array}{c}\text { Sporadic, } n=105 \\
(38.3)\end{array}$ & $\begin{array}{c}\text { NF1-Assoc, } n=143 \\
(52.2)\end{array}$ & $\begin{array}{l}\text { Radiation-Assoc, } \\
\qquad n=26(9.5)\end{array}$ & \\
\hline \multicolumn{6}{|l|}{ Surgery } \\
\hline Y & $259(94.5)$ & $102(97.1)$ & $133(93.0)$ & $24(92.3)$ & $0.321(0.248)$ \\
\hline $\mathrm{N}$ & $15(5.5)$ & $3(2.9)$ & $10(7.0)$ & $2(92.3)$ & \\
\hline \multicolumn{6}{|l|}{ Resection Margins ( $n=259$ ) } \\
\hline R0 & $145(60.0)$ & $66(64.7)$ & $70(52.6)$ & $9(37.5)$ & $<0.001 \ddagger(<0.001)$ \\
\hline $\mathrm{R} 1$ & $54(20.8)$ & $13(12.7)$ & $36(27.1)$ & $5(20.8)$ & \\
\hline $\mathrm{R} 2$ & $23(8.9)$ & $7(6.9)$ & $9(6.8)$ & $7(29.2)$ & \\
\hline Unknown & $37(14.3)$ & $16(15.7)$ & $18(13.5)$ & $3(12.5)$ & \\
\hline Therapy, resected $(n=259)$ & & & & & $0.312 \S(0.590)$ \\
\hline Surgery alone & $75(27.3)$ & $31(29.5)$ & $36(25.2)$ & $8(30.8)$ & \\
\hline Chemotherapy alone + surgery & $45(16.4)$ & $19(18.1)$ & $19(13.3)$ & $7(26.9)$ & \\
\hline Neoadjuvant & $18(6.6)$ & $5(4.7)$ & $10(6.9)$ & $3(11.5)$ & \\
\hline Adjuvant & $26(9.5)$ & $14(13.3)$ & $9(6.3)$ & $3(11.5)$ & \\
\hline Both neo-/adjuvant & $1(0.3)$ & $0(0.0)$ & $0(0.0)$ & $1(3.8)$ & \\
\hline Radiation alone + surgery & $75(27.4)$ & $29(27.6)$ & $39(27.3)$ & $7(26.9)$ & \\
\hline Neoadjuvant & $11(4.0)$ & $3(2.8)$ & $8(5.9)$ & $0(0.0)$ & \\
\hline Adjuvant & $63(22.9)$ & $26(24.8)$ & $31(21.7)$ & $6(23.1)$ & \\
\hline Other (IORT) & $1(0.3)$ & $0(0.0)$ & $0(0.0)$ & $1(3.8)$ & \\
\hline Chemoradiotherapy + surgery & $64(24.4)$ & $23(21.9)$ & $39(27.3)$ & $2(7.7)$ & \\
\hline Neoadjuvant & $22(8.0)$ & $8(7.6)$ & $14(9.8)$ & $0(0.0)$ & \\
\hline Adjuvant & $29(10.6)$ & $14(13.3)$ & $14(9.8)$ & $1(3.8)$ & \\
\hline Other combination & $13(4.7)$ & $1(1.0)$ & $11(7.7)$ & $1(3.8)$ & \\
\hline Palliative therapy, no resection $(n=15)$ & & & & & $0.240(0.296)$ \\
\hline Chemo & $9(3.3)$ & $3(2.9)$ & $5(3.5)$ & $1(3.8)$ & \\
\hline Radiotherapy & $1(0.3)$ & $0(0.0)$ & $1(0.6)$ & $0(0.0)$ & \\
\hline Chemoradiotherapy & $5(1.8)$ & $0(0.0)$ & $4(2.7)$ & $1(3.8)$ & \\
\hline LR & $98(35.7)$ & $33(31.4)$ & $51(35.7)$ & $14(53.8)$ & $0.102(0.486)$ \\
\hline DR & $120(43.8)$ & $47(44.8)$ & $65(45.5)$ & $8(30.8)$ & $0.369(0.914)$ \\
\hline
\end{tabular}

Chemo = chemotherapy; $\mathrm{DR}=$ distant recurrence; IORT = intraoperative radiotherapy; LR = local recurrence; $N=$ no; $Y=$ yes.

${ }^{*}$ Values are number (\%). The sum of percentages may not equal 100 due to rounding. Boldface type indicates statistical significance of $p<0.05$.

$\dagger$ Comparison between sporadic, NF1-associated, and radiation-associated characteristics (comparison between sporadic and NF1-associated characteristics).

$\ddagger$ Comparisons exclude unknown resection margins.

$\S$ Overall comparison between resection alone, chemotherapy + surgery, radiotherapy + surgery, or chemoradiotherapy + surgery.

as, in the vast majority of cases, they cannot be meaningfully irradiated without unacceptable toxicity. Therefore, an alternative local relapse outcome analysis including patients who were treated at our institution for their primary sporadic or NF1-associated disease was constructed to evaluate the utility of radiation therapy in this group (Table 5). In univariate analysis we observed a local recurrence risk reduction in patients who received radiation therapy and resection $(p=0.058$, HR 0.49$)$. The 5 - and 10 year LRFS for patients who received radiation therapy was $82 \%$ and $71 \%$, respectively, versus $67 \%$ and $59 \%$, respectively, for those who did not. Adjusted multivariate analyses for this cohort revealed positive margins and males to be associated with worse LRFS.

Five-year DRFS was 50\%. Adverse predictors of DRFS in univariate analysis included male sex, nonepithelioid or triton MPNSTs, tumors $\geq 10 \mathrm{~cm}$, deep location, and treatment with chemotherapeutics (Table 4). Only tumor size ( $\geq$ $10 \mathrm{~cm}$ ) remained a significant predictor of poor DRFS in multivariate analysis $(p=0.034$, HR 1.76 ; Table 4$)$.

\section{High-Grade MPNST Disease-Specific Survival}

Of the entire cohort $(n=289), 131$ patients died of disease, 17 died of unrelated causes, 86 had no evidence of active malignancy, and 55 were alive with disease at the end of the follow-up period. Median DSS time was 5.5 years, and 5-year DSS was $51 \%$ (Fig. 2). Patients with high-grade MPNST who had incomplete $(\mathrm{R} 2)$ resection $(\mathrm{n}=23)$, did not receive surgical intervention $(n=22)$, or had metastasis at presentation $(n=15)$ had similar outcomes with significantly worse prognosis than patients who presented with localized disease and received $\mathrm{R} 0$ or $\mathrm{R} 1$ resection; there- 
TABLE 3. Characteristics and survival in 12 patients with low-grade MPNSTs

\begin{tabular}{|c|c|c|c|c|c|c|c|c|c|c|}
\hline $\begin{array}{l}\text { Case } \\
\text { No. }\end{array}$ & Subtype & $\begin{array}{l}\text { Age } \\
\text { (yrs) }\end{array}$ & Location & $\begin{array}{c}\text { Neurofibroma } \\
\text { Component }\end{array}$ & Depth & Therapy & $\begin{array}{l}\text { Surgical } \\
\text { Margins }\end{array}$ & LR & DR & DOD \\
\hline 1 & NF1-assoc & 26 & Thoracic spine & Spinal & $D$ & None & R0 & $\mathrm{N}$ & $\mathrm{N}$ & $\mathrm{N}$ \\
\hline 2 & NF1-assoc & 39 & Intercostal nerve & Plexiform & $\mathrm{D}$ & None & Ro & $\mathrm{N}$ & $\mathrm{N}$ & $\mathrm{N}$ \\
\hline 3 & NF1-assoc & 47 & Sciatic nerve, thigh & Diffuse & $D$ & None & Ro & $\mathrm{N}$ & $\mathrm{N}$ & $\mathrm{N}$ \\
\hline 4 & NF1-assoc & 43 & Brachial plexus & Plexiform & $D$ & None & $\mathrm{R} 1$ & $\mathrm{~N}$ & $\mathrm{~N}$ & $\mathrm{~N}$ \\
\hline 5 & NF1-assoc & 69 & Back & Plexiform & $S$ & Radiotherapy & $\mathrm{R} 1$ & $\mathrm{~N}$ & $\mathrm{~N}$ & $\mathrm{~N}$ \\
\hline 6 & Radiation-assoc & 60 & Brachial plexus & None & $\mathrm{D}$ & None & $\mathrm{R} 2$ & Y & $\mathrm{N}$ & $\mathrm{N}$ \\
\hline 7 & Sporadic & 68 & Chest & None & $S$ & Radiotherapy & R0 & $\mathrm{N}$ & $\mathrm{N}$ & $\mathrm{N}$ \\
\hline 8 & Sporadic & 13 & Forehead & None & $S$ & None & R1 & Y & $\mathrm{N}$ & $\mathrm{N}$ \\
\hline 9 & Sporadic & 60 & Lumbosacral & None & $D$ & Chemo & Ro & $Y$ & $\mathrm{~N}$ & $\mathrm{~N}$ \\
\hline 10 & Sporadic & 40 & Peritoneum & None & $D$ & None & Ro & $\mathrm{N}$ & $\mathrm{N}$ & $\mathrm{N}$ \\
\hline 11 & Sporadic & 64 & Shoulder & None & $S$ & None & Ro & $\mathrm{N}$ & $\mathrm{N}$ & $\mathrm{N}$ \\
\hline 12 & Sporadic & 79 & Retroperitoneum & None & $\mathrm{D}$ & Chemo & Ro & $\mathrm{N}$ & $\mathrm{N}$ & $\mathrm{N}$ \\
\hline
\end{tabular}

DOD = MPNST-specific death.

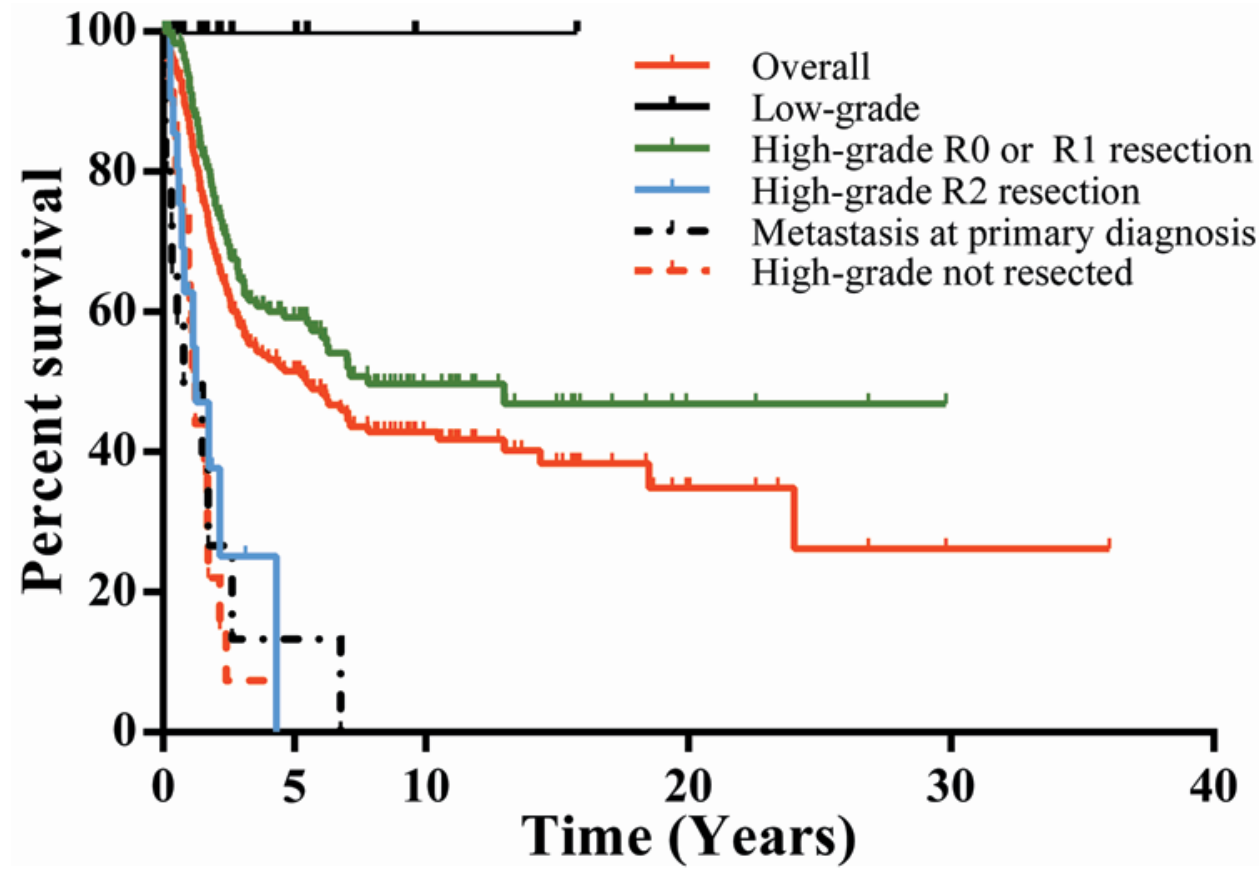

\begin{tabular}{llll}
\hline Survival Estimates & 2-year & 5-year & 10-year \\
\hline Overall & $68 \%$ & $52 \%$ & $42 \%$ \\
Low-grade & $100 \%$ & $100 \%$ & $100 \%$ \\
High-grade R0 or R1 resection & $75 \%$ & $59 \%$ & $49 \%$ \\
High-grade R2 resection & $37 \%$ & $0 \%$ & $0 \%$ \\
High-grade not resected & $22 \%$ & - & - \\
Metastasis at primary diagnosis & $27 \%$ & $13 \%$ & $0 \%$
\end{tabular}

FIG. 2. Kaplan-Meier curves showing DSS for the MPNST populations. 


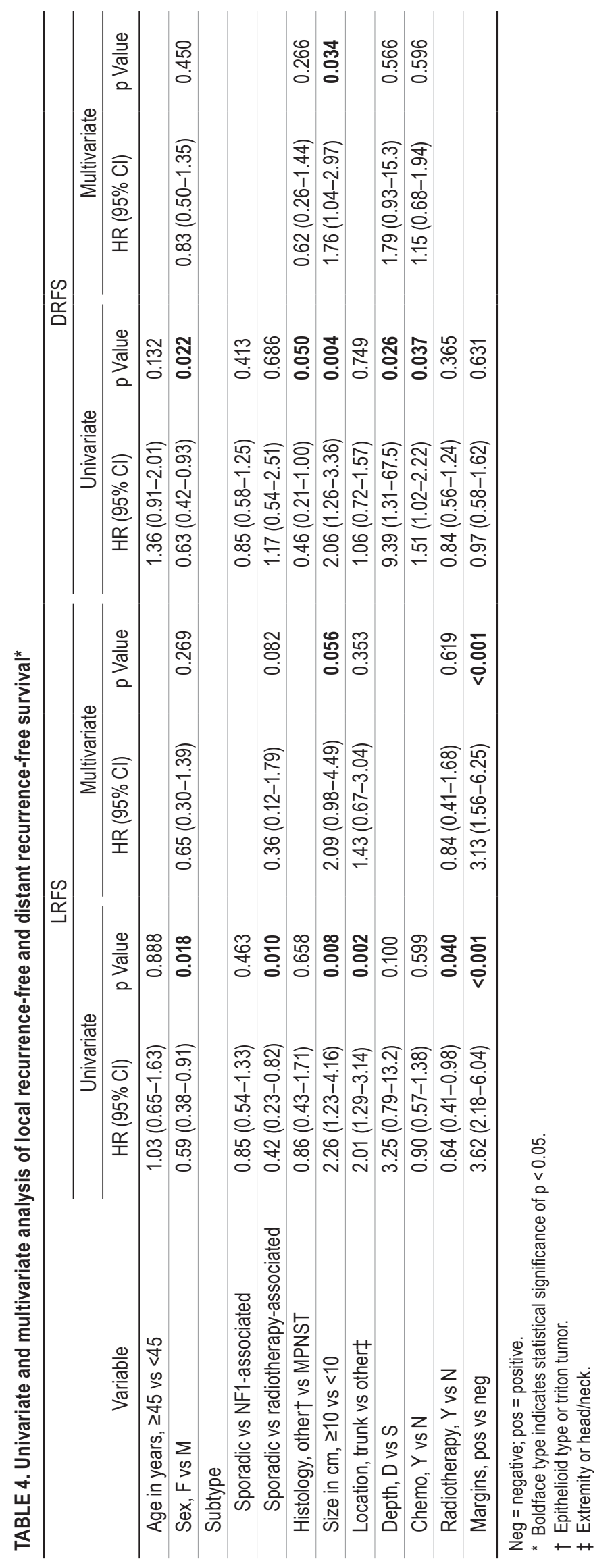

fore, these patients were excluded from subsequent univariate and multivariate survival analyses. Survival was worse for patients who underwent amputation compared with those who underwent R0 limb-sparing surgery ( $\mathrm{p}=$ 0.003 , HR 4.5, 5-year DSS 25\% and 78\%, respectively).

Individual interrogation between MPNST subtypes revealed superior DSS in sporadic compared with NF1-associated and radiation-associated patients $(\mathrm{p}=0.016, \mathrm{HR}$ $0.59 ; p=0.058$, HR 0.52; Table 6, Fig. 3). Truncal location, positive resection margins, local recurrence, and metastasis were adverse predictors of DSS in multivariate analysis $(\mathrm{p}=0.039$, HR 1.92; $\mathrm{p}=0.014$, HR 2.41; $\mathrm{p}=0.059, \mathrm{HR}$ $1.95 ; \mathrm{p}<0.001$, HR 10.7, respectively).

\section{Discussion}

In the present study, we aimed to identify and contrast features predictive of recurrence and survival in patients with sporadic, NF1-associated, and radiation-associated MPNST. First, we found that low-grade MPNSTs have a benign natural history, with a low risk of recurrence with negative margin resection and better survival outcomes compared with their high-grade counterparts. In our investigation of high-grade MPNST, the importance of negative margin resection was confirmed to be a key determinant of local control and disease-specific outcomes. We found that local recurrence patterns are more aggressive in radiation-associated MPNST compared with sporadic or NF1-associated MPNST, which may in part be attributed to the propensity for these tumors to be deeply located in the trunk, where negative margins were less likely to be achieved. Lower survival estimates in NF1- and radiationassociated MPNST may in part be explained by their differences in location, size, and positive margin rate compared with sporadic MPNST.

A review of 6 recent similar single-institution retrospective series that had at minimum 100 patients in their cohort is summarized in Table 7. Including our series, the most common adverse prognostic factor associated with survival was large tumor size ( 6 of 7 studies), followed by positive resection margins ( 3 of 7 studies) and truncal location (2 of 7). Only Porter et al. ${ }^{19}$ identified NF1 as an adverse prognostic factor associated with survival while Stucky et al. ${ }^{23}$ and our study identified a trend. In our cohort, a shorter disease-specific survival trend for radiation-associated MPNST was noted when compared with sporadic and NF1-associated MPNST, which supports findings by LaFemina et al. ${ }^{15}$ in which radiation-associated MPNST patients were found to have a poorer prognosis. Six of the 7 series investigated the impact of chemotherapy and radiotherapy on survival outcomes, and only Anghileri et al. ${ }^{1}$ reported radiation therapy to be associated with a significant survival benefit.

MPNSTs pose a challenging management dilemma. $\mathrm{Pa}-$ tients often present with large tumors that infiltrate multiple segments of nerve, which makes obtaining negative margins and maintaining acceptable neural functionality with surgical intervention problematic. Similar to others, we found that patients who underwent resection with negative margins and tumors $<10 \mathrm{~cm}$ had better LRFS outcomes..$^{6,8,10}$ Additionally, our study demonstrates that 

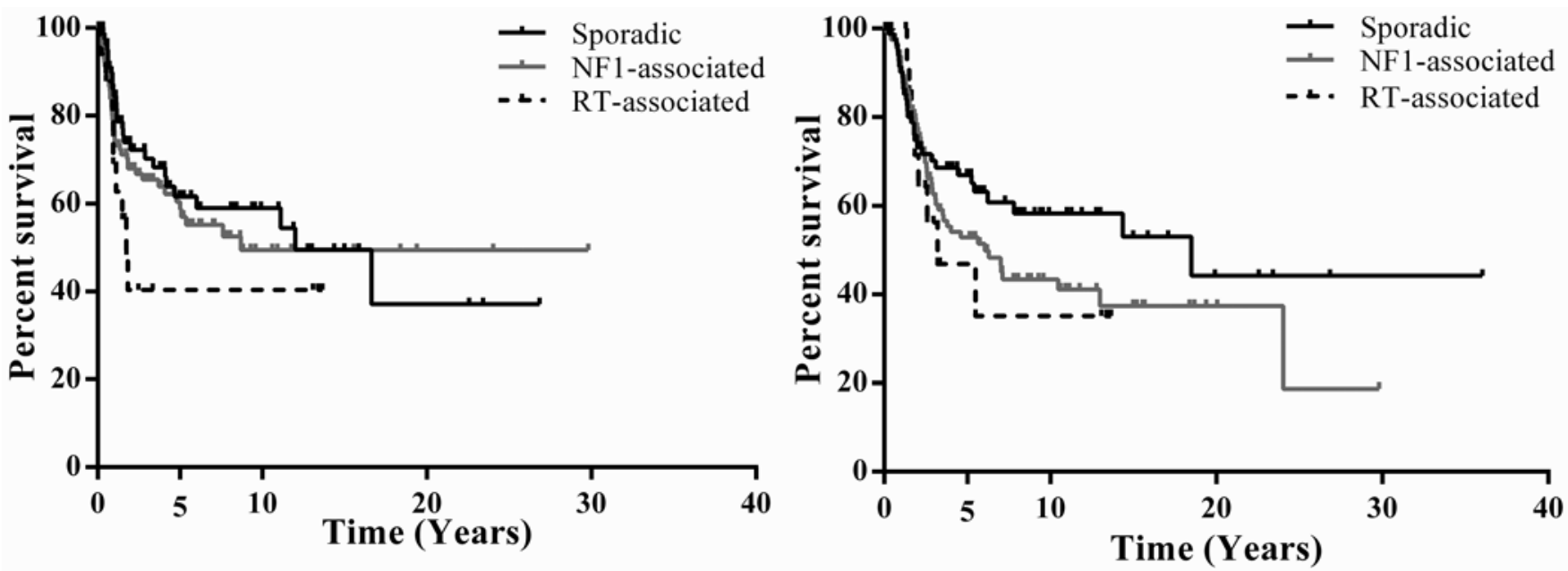

FIG. 3. Kaplan-Meier curves showing LRFS stratified by MPNST subtype (RT-associated vs sporadic, $p=0.010$; RT-associated vs NF1-associated, $p=0.232$ ) (left), and DSS stratified by MPNST subtype (RT-associated vs sporadic, $p=0.272$; RT-associated vs NF1-associated, $p=0.714$ ) (right). RT-associated $=$ radiotherapy-associated.

radiotherapy in combination with an $\mathrm{R} 0 / \mathrm{R} 1$ resection is associated with better local control (5-year LRFS $82 \%$ vs $67 \%$ for those who were not treated with radiation therapy). Despite this finding, the use of radiation therapy failed to definitively show benefits associated with disease-specific outcomes (similar to previous reports). ${ }^{1,7,19,23,25}$

Current chemotherapeutics have not been consistently shown to provide a significant survival benefit. ${ }^{1,719,23,25} \mathrm{We}$ found that patients treated with chemotherapy had worse outcomes than those who did not receive chemotherapy. However, in our cohort, chemotherapy was administered more often to patients with truncally located or large tumors and to patients with advanced disease. The therapeutic benefits of chemotherapy, therefore, remain unclear. Three patients had complete response to therapy, which illustrates that chemotherapy can be of significant benefit in select patients. Additional research is needed to identify predictive biomarkers for therapeutic response to improve outcomes for patients with MPNST.
Cytogenetic analysis and DNA sequencing of MPNSTs has identified several recurring genetic aberrations (involving genes such as NF1, TP53, CDKN1B, PDGFRA, and $H G F$ ) and irregular receptor tyrosine kinase activity that could be exploited for treatment or to highlight other targetable molecular dysregulations. ${ }^{12,17,20}$ However, several clinical trials using the targeted inhibitors erlotinib, sorafenib, and imatinib, among others, have been conducted in sarcoma patients, including those with MPNST, with minimal observed responses. ${ }^{3,8}$ Combination therapy to achieve a more complete signaling blockade, such as coinhibition of signaling pathways and upstream activators; the conversion of promising preclinical targets into clinical trials, such as histone deacetylase inhibitors and PI3K/mTOR inhibitors; or further identification of targetable nodes, may improve outcomes for MPNST patients. ${ }^{13,16,26}$

Our study is limited by its retrospective nature, the inherent heterogeneity of primary management, and the

TABLE 5. Univariate and multivariate predictors of adverse LRFS in patients with sporadic or NF1-asscociated MPNST who presented to our institution with primary disease $(n=128)^{*}$

\begin{tabular}{|c|c|c|c|c|}
\hline \multirow[b]{2}{*}{ Variable } & \multicolumn{2}{|c|}{ Univariate } & \multicolumn{2}{|c|}{ Multivariate } \\
\hline & $\mathrm{HR}(95 \% \mathrm{Cl})$ & $p$ Value & $\mathrm{HR}(95 \% \mathrm{Cl})$ & p Value \\
\hline Age in years, $\geq 45$ vs $<45$ & $0.85(0.38-1.91)$ & 0.691 & & \\
\hline Sex, F vs M & $0.37(0.17-0.82)$ & 0.014 & $0.34(0.12-0.94)$ & 0.039 \\
\hline Subtype, sporadic vs NF1-associated & $0.40(0.17-0.84)$ & 0.036 & $0.38(0.13-1.15)$ & 0.385 \\
\hline Histology, other† vs MPNST & $0.67(0.34-2.32)$ & 0.805 & & \\
\hline Size in $\mathrm{cm}, \geq 10$ vs $<10$ & $2.35(0.92-5.99)$ & 0.074 & & \\
\hline Location, trunk vs other $\ddagger$ & $2.06(0.99-4.27)$ & 0.051 & $1.17(0.48-2.84)$ & 0.721 \\
\hline Chemo, $Y(n=59)$ vs $N(n=69)$ & $1.06(0.52-2.19)$ & 0.862 & & \\
\hline Radiotherapy, Y ( $n=69)$ vs N $(n=59)$ & $0.49(0.24-1.02)$ & 0.058 & $0.49(0.20-1.23)$ & 0.130 \\
\hline Margins, pos vs neg & $5.08(2.27-11.4)$ & $<0.001$ & $3.90(1.69-8.97)$ & 0.001 \\
\hline
\end{tabular}

* Patients with localized primary sporadic or NF1-associated MPNST who underwent R0 or R1 resection. Boldface type indicates statistical significance of $p<0.05$.

$\dagger$ Epithelioid type or triton tumor.

‡ Extremity or head/neck. 
TABLE 6. Univariate and multivariate predictors of adverse disease-specific survival*

\begin{tabular}{|c|c|c|c|c|}
\hline \multirow[b]{2}{*}{ Variables } & \multicolumn{2}{|c|}{ Univariate } & \multicolumn{2}{|c|}{ Multivariate } \\
\hline & $\operatorname{HR}(95 \% \mathrm{Cl})$ & $p$ Value & $\mathrm{HR}(95 \% \mathrm{Cl})$ & $\mathrm{p}$ Value \\
\hline Age in years, $\geq 45$ vs $<45$ & $1.18(0.77-1.81)$ & 0.438 & & \\
\hline Sex, F vs M & $0.66(0.43-0.99)$ & 0.047 & $1.53(0.81-2.91)$ & 0.194 \\
\hline \multicolumn{5}{|l|}{ Subtype } \\
\hline Sporadic vs NF1-associated & $0.59(0.39-0.91)$ & 0.016 & $1.00(0.48-2.06)$ & 0.997 \\
\hline Sporadic vs radiotherapy-associated & $0.52(0.26-1.08)$ & 0.058 & $1.35(0.48-3.75)$ & 0.568 \\
\hline Histology, other† vs MPNST & $0.59(0.27-1.29)$ & 0.192 & & \\
\hline Size in $\mathrm{cm}, \geq 10$ vs $<10$ & $3.14(1.88-5.23)$ & $<0.001$ & $1.78(0.91-3.84)$ & 0.092 \\
\hline Location, trunk vs other $\ddagger$ & $1.56(1.07-2.27)$ & 0.021 & $1.92(1.03-3.58)$ & 0.039 \\
\hline Depth, D vs S & $2.37(0.75-7.52)$ & 0.141 & & \\
\hline Chemo, Y vs N & $1.68(1.12-2.53)$ & 0.012 & $1.46(0.76-2.08)$ & 0.261 \\
\hline Radiotherapy, Y vs N & $0.64(0.43-0.97)$ & 0.035 & $0.81(0.46-1.47)$ & 0.501 \\
\hline Margins, pos vs neg & $2.14(1.32-3.45)$ & 0.002 & $2.41(1.19-4.84)$ & 0.014 \\
\hline LR, Y vs N & $2.46(1.63-3.72)$ & $<0.001$ & $1.95(0.96-3.89)$ & 0.059 \\
\hline $\mathrm{DR}, \mathrm{Y}$ vs $\mathrm{N}$ & $4.61(2.83-7.52)$ & $<0.001$ & $10.7(4.89-23.5)$ & $<0.001$ \\
\hline
\end{tabular}

small number of radiation-associated MPNSTs meeting study eligibility criteria. Furthermore, as a high-volume tertiary center, we were able to obtain, to our knowledge, the largest single-institution MPNST cohort to date, but follow-up data are variable between survivors. However, our cohort was subjected to thorough subset analysis and offers insights on the management of a rare disease that is understudied as a result of limited cohort sizes. Few studies reporting the natural history of MPNST have described the features and outcomes of these aggressive tumors with specific attention to MPNST etiology as exhaustively as the present series. In addition to reporting the treatment and outcomes for our cohort, we profile the nerves of involvement and accompanying symptoms with respect to the MPNST subtype, profile the sites of metastasis, and describe the behavior of low-grade MPNSTs separately from high-grade MPNSTs.

\section{Conclusions}

The goal of curative management for all MPNSTs should be negative margin resection whenever possible. Additionally, supplemental radiation therapy for local control should be considered. Large index tumor size was implicated as a major adverse prognostic factor, which recapitulates the importance of early diagnosis and intervention in these aggressive tumors, particularly in the NF1 population where fluorine-18-labeled fluorodeoxyglucose PET/CT has been shown to be a useful adjunct imaging tool to MRI when malignant change needs to be excluded. ${ }^{5,27}$ Finally, differences in clinicopathological features and patterns of local recurrence and survival do exist between sporadic, NF1-asssociated, and radiation-associated MPNSTs; therefore, the etiology of these tumors should be considered in the management of patients and in future studies evaluating tumor biology.

TABLE 7. A review of MPNST in select recent single-institution retrospective studies

\begin{tabular}{|c|c|c|c|c|c|c|c|c|c|}
\hline \multirow[b]{2}{*}{ Authors \& Year } & \multirow{2}{*}{$\begin{array}{l}\text { No. of } \\
\text { Pts }\end{array}$} & \multirow{2}{*}{$\begin{array}{l}\% 5 \text {-Year } \\
\text { Survival }\end{array}$} & \multicolumn{4}{|c|}{ Adverse Prognostic Features } & \multirow{2}{*}{$\begin{array}{c}\text { Pos } \\
\text { Margins }\end{array}$} & \multicolumn{2}{|c|}{ Therapy Benefit } \\
\hline & & & NF1-Assoc & Radiation-Assoc & Location & Size & & Radiotherapy & Chemo \\
\hline Present series & 289 & $52 \dagger$ & $\mathrm{T}$ & NS & $\mathrm{T}$ (trunk) & $S S(\geq 10 \mathrm{~cm})$ & SS & NS & NS \\
\hline LaFemina et al., 2013 & 105 & - & NS & SS & NS & SS (larger) & SS & - & - \\
\hline Fan et al., 2014 & 146 & $57 \ddagger$ & NS & - & NS & NS & NS & NS & NS \\
\hline Stucky et al., $2012 \S$ & 175 & $60 \dagger$ & $\mathrm{T}$ & - & SS (trunk) & $\mathrm{SS}(\geq 5 \mathrm{~cm})$ & NS & NS & NS \\
\hline Zou et al., 2009 & 140 & $39 \dagger$ & NS & NS & NS & $S S(\geq 10 \mathrm{~cm})$ & NS & NS & NS \\
\hline Porter et al., 2009 & 123 & $51 \ddagger$ & SS & - & NS & SS (>200 ml) & NS & NS & NS \\
\hline Anghileri et al., 2006 & 205 & $40 \dagger$ & NS & - & SS (trunk, HN) & SS (larger) & SS & SS & NS \\
\hline
\end{tabular}

$\mathrm{HN}=$ head/neck; NS = not statistically significant; pts = patients; $\mathrm{SS}=$ statistically significant; $\mathrm{T}=$ trend.

$\dagger$ Disease-specific survival.

$\ddagger$ Overall survival.

$\S$ Series was a single-institution study but patients were treated at 3 different locations. 


\section{Acknowledgments}

Funding for this research was provided in part by NIH/ NCI K08CA160443 (K.E.T.), The Sally M. Kingsbury Sarcoma Research Foundation (K.E.T.), and Amschwand Sarcoma Cancer Foundation (supporting C.M.K.). We thank Jill Designe for review and editing the manuscript. We also thank Geoffrey Giacco for identification of the study cohort.

\section{References}

1. Anghileri M, Miceli R, Fiore M, Mariani L, Ferrari A, Mussi $\mathrm{C}$, et al: Malignant peripheral nerve sheath tumors: prognostic factors and survival in a series of patients treated at a single institution. Cancer 107:1065-1074, 2006

2. Bernthal NM, Putnam A, Jones KB, Viskochil D, Randall RL: The effect of surgical margins on outcomes for low grade MPNSTs and atypical neurofibroma. J Surg Oncol 110:813-816, 2014

3. Bradford D, Kim A: Current treatment options for malignant peripheral nerve sheath tumors. Curr Treat Options Oncol 16:328, 2015

4. Brennan MF, Antonescu CR, Moraco N, Singer S: Lessons learned from the study of 10,000 patients with soft tissue sarcoma. Ann Surg 260:416-422, 2014

5. Chirindel A, Chaudhry M, Blakeley JO, Wahl R: ${ }^{18} \mathrm{~F}-\mathrm{FDG}$ $\mathrm{PET} / \mathrm{CT}$ qualitative and quantitative evaluation in neurofibromatosis type 1 patients for detection of malignant transformation: comparison of early to delayed imaging with and without liver activity normalization. J Nucl Med 56:379_ 385, 2015

6. Ducatman BS, Scheithauer BW, Piepgras DG, Reiman HM, Ilstrup DM: Malignant peripheral nerve sheath tumors. A clinicopathologic study of 120 cases. Cancer 57:2006-2021, 1986

7. Fan Q, Yang J, Wang G: Clinical and molecular prognostic predictors of malignant peripheral nerve sheath tumor. Clin Transl Oncol 16:191-199, 2014

8. Farid M, Demicco EG, Garcia R, Ahn L, Merola PR, Cioffi A, et al: Malignant peripheral nerve sheath tumors. Oncologist 19:193-201, 2014

9. Fletcher CDM, Bridge JA, Hogendoorn P, Mertens F, eds: WHO Classification of Tumours of Soft Tissue and Bone. Lyon, France: IARC, 2013

10. Gladdy RA, Qin LX, Moraco N, Edgar MA, Antonescu CR, Alektiar KM, et al: Do radiation-associated soft tissue sarcomas have the same prognosis as sporadic soft tissue sarcomas? J Clin Oncol 28:2064-2069, 2010

11. Grobmyer SR, Reith JD, Shahlaee A, Bush CH, Hochwald SN: Malignant peripheral nerve sheath tumor: molecular pathogenesis and current management considerations. J Surg Oncol 97:340-349, 2008

12. Jhanwar SC, Chen Q, Li FP, Brennan MF, Woodruff JM: Cytogenetic analysis of soft tissue sarcomas. Recurrent chromosome abnormalities in malignant peripheral nerve sheath tumors (MPNST). Cancer Genet Cytogenet 78:138-144, 1994

13. Johansson G, Mahller YY, Collins MH, Kim MO, Nobukuni $\mathrm{T}$, Perentesis J, et al: Effective in vivo targeting of the mammalian target of rapamycin pathway in malignant peripheral nerve sheath tumors. Mol Cancer Ther 7:1237-1245, 2008

14. Kolberg M, Høland M, Agesen TH, Brekke HR, Liestøl K, Hall KS, et al: Survival meta-analyses for $>1800$ malignant peripheral nerve sheath tumor patients with and without neurofibromatosis type 1. Neuro Oncol 15:135-147, 2013

15. LaFemina J, Qin LX, Moraco NH, Antonescu CR, Fields RC, Crago AM, et al: Oncologic outcomes of sporadic, neurofibromatosis-associated, and radiation-induced malignant peripheral nerve sheath tumors. Ann Surg Oncol 20:66-72, 2013
16. Lopez G, Torres K, Liu J, Hernandez B, Young E, Belousov $\mathrm{R}$, et al: Autophagic survival in resistance to histone deacetylase inhibitors: novel strategies to treat malignant peripheral nerve sheath tumors. Cancer Res 71:185-196, 2011

17. Mertens F, Dal Cin P, De Wever I, Fletcher CDM, Mandahl N, Mitelman F, et al: Cytogenetic characterization of peripheral nerve sheath tumours: a report of the CHAMP study group. J Pathol 190:31-38, 2000

18. Neurofibromatosis. Natl Inst Health Consens Dev Conf Consens Statement 6:1-7, 1987

19. Porter DE, Prasad V, Foster L, Dall GF, Birch R, Grimer RJ: Survival in malignant peripheral nerve sheath tumours: a comparison between sporadic and neurofibromatosis Type 1-associated tumours. Sarcoma 2009:756395, 2009

20. Rahrmann EP, Watson AL, Keng VW, Choi K, Moriarity BS, Beckmann DA, et al: Forward genetic screen for malignant peripheral nerve sheath tumor formation identifies new genes and pathways driving tumorigenesis. Nat Genet 45:756-766, 2013

21. Rodriguez FJ, Folpe AL, Giannini C, Perry A: Pathology of peripheral nerve sheath tumors: diagnostic overview and update on selected diagnostic problems. Acta Neuropathol 123:295-319, 2012

22. Schaefer IM, Fletcher CD: Malignant peripheral nerve sheath tumor (MPNST) arising in diffuse-type neurofibroma: clinicopathologic characterization in a series of 9 cases. Am J Surg Pathol 39:1234-1241, 2015

23. Stucky CC, Johnson KN, Gray RJ, Pockaj BA, Ocal IT, Rose PS, et al: Malignant peripheral nerve sheath tumors (MPNST): the Mayo Clinic experience. Ann Surg Oncol 19:878-885, 2012

24. Weiss SW, Goldblum JR: Malignant tumors of the peripheral nerves, in Enzinger and Weiss's Soft Tissue Tumors, ed 5. St. Louis: Mosby Elsevier, 2008, pp 903-925

25. Zou C, Smith KD, Liu J, Lahat G, Myers S, Wang WL, et al: Clinical, pathological, and molecular variables predictive of malignant peripheral nerve sheath tumor outcome. Ann Surg 249:1014-1022, 2009

26. Zou CY, Smith KD, Zhu QS, Liu J, McCutcheon IE, Slopis $\mathrm{JM}$, et al: Dual targeting of AKT and mammalian target of rapamycin: a potential therapeutic approach for malignant peripheral nerve sheath tumor. Mol Cancer Ther 8:11571168,2009

27. Khiewvan B, Macapinlac HA, Lev D, McCutcheon IE, Slopis JM, Al Sannaa G, et al: The value of ${ }^{18} \mathrm{~F}-\mathrm{FDG}$ PET/CT in the management of malignant peripheral nerve sheath tumors. Eur J Nucl Med Mol Imaging 41:1756-1766, 2014

\section{Disclosures}

Dr. Hunt reports being a consultant with Armada Health.

\section{Author Contributions}

Conception and design: Torres, Watson, Al Sannaa, Lazar. Acquisition of data: Torres, Watson. Analysis and interpretation of data: all authors. Drafting the article: Torres, Watson. Critically revising the article: all authors. Reviewed submitted version of manuscript: all authors. Approved the final version of the manuscript on behalf of all authors: Torres. Statistical analysis: Watson. Administrative/technical/material support: Torres. Study supervision: Torres.

\section{Correspondence}

Keila E. Torres, Department of Surgical Oncology, 1400 Pressler St., Unit 1484, Houston, TX 77030. email: ketorres@ mdanderson.org. 